\title{
Isolation of Mycobacteriophage: Novel tool to treat Mycobacterium spp
}

\author{
R. Satish ${ }^{1^{*}}$, A. Desouza ${ }^{2}$. \\ ${ }^{1}$ Department of Microbiology, SIES College of Arts, Science \&Commerce, Sion(west), Mumbai University, Mumbai, India. \\ ${ }^{2}$ Department of Microbiology, SIES College of Arts, Science \&Commerce, Sion(west), Mumbai University, Mumbai, India. \\ *Corresponding Author: rajithasatishb@gmail.com,Tel.91-9833716190
}

Available online at: www.isroset.org

Received: 21/Mar/2018, Revised: 05/Apr/2018, Accepted: 23/Apr/2018, Online: 30/Apr/ 2018

\begin{abstract}
Mycobacteriophages are viruses that infect Mycobacterium spp. To date, 9666 mycobacteriophages have been isolated and 1519 mycobacteriophage genomes have been sequenced(phagesdb.org). The aim of this study was to isolate mycobacteriophages from different soil samples using Mycobacterium smegmatis as host. In this study mycobacteriophages have been isolated from 10 different soil samples. Presence of these phages was confirmed by qualitative plaque formation on plates. These 10 different phages were further tested for host diversity using M. fortuitum subsp. fortuitum MTCC993, Mycobacterium kansasiiMTCC3058, Mycobacterium aviumsubsp. avium MTCC1723 and Mycobacterium tuberculosis MTCC300. Three among these 10 phages, were found to infect all the 4 different species of Mycobacterium besides the host Mycobacterium smegmatis that was used for isolation of the phages. This truly reflects the host diversity of the phages and their ability to rapidly adapt to new hosts. These phages could also hold a great potential to be used as tools of genetic manipulation to study Mycobacteria. Their potential for the treatment and eradication of M. tuberculosis can also be studied further.
\end{abstract}

Keywords-Mycobacteriophages, Mycobacterium tuberculosis, Mycobacterium smegmatis, MDR-TB, Host diversity.

\section{INTRODUCTION}

Tuberculosis is the ninth leading cause of death worldwide and the leading cause due to a single infectious agent, ranking above HIV/AIDS. In 2016, there were an estimated 1.3 million TB deaths among HIV-negative people and an additional 3,74,000 deaths among HIV-positive people [website1].

According to the WHO, Annual TB report for South East Asia 2017 ,TB which is one of the oldest diseases is still one of the biggest killers, with a high morbidity rate, especially in South East Asian Region where India alone accounts for one third of the global burden i.e $23 \%$.

MDR-TB still remains a public health crisis. In 2016, WHO estimated that there were 600,000 new cases with resistance to rifampicin - the most effective first-line drug of which 490,000 had MDR-TB. The MDR-TB burden largely falls on 3 countries - India, China and the Russian Federation - which together account for nearly half of the global cases. About $6.2 \%$ of MDR-TB cases had XDR-TB in 2016 [website 2].

The remarkable fact is that global control of tuberculosis, a disease that kills someone every 20 seconds, depends upon a 125 -year-old test, a 85-year-old vaccine and drugs that take six months to cure and have not been changed in four decades. To successfully treat tuberculosis and prevent resistance, we need to use better tools and accelerate the development of new tools for the future. We need new drugs, vaccines and diagnostics, as well as innovations in tuberculosis control and case management [1].

Specific targets set by WHO in the "End TB Strategy" include a $90 \%$ reduction in TB deaths and an $80 \%$ reduction in TB incidence (new cases per year) by 2030, as compared to the number of TB cases in 2015. Achieving these targets requires improvising TB care and prevention with new diagnostics, drugs, treatment regimens and vaccines [website 3].

The rapid spread of drug-resistant tuberculosis and isolation of Mycobacterium tuberculosis (MTB) resistant to the first line drugs isoniazid and rifampicin (multidrug-resistant strains) and to the second and third line drugs (extensively drug-resistant strains) has attracted much interest in mycobacteriophages. This interest, in addition to fundamental studies of the evolution and diversity of a large number of mycobacteriophages due to the advent of DNA sequencing has helped in greater understanding of mycobacterial physiology and pathogenesis mechanisms. Renewed interest in mycobacteriophages is also due to the possibility of therapeutic application of mycobacteriophages with lytic properties against virulent MTB species [2]. 
Bacteriophages are the most numerous biological entities in the biosphere, and their genetic diversity is high. However bacteriophages including Mycobacteriophages remain ill defined.[3] Mycobacteriophages are viruses that infect mycobacterial hosts, such as Mycobacterium tuberculosis and Mycobacterium smegmatis[4].Mycobacteriophage are generally composed of double stranded DNA surrounded by a protein capsid. Their chromosomes exhibit great morphological and genetic diversity [5]. To date, 9666 mycobacteriophages have been isolated and 1519 mycobacteriophage genomes have been sequenced (website 4). Mycobacteriophages have been used in the construction of tools for genetic manipulation of Mycobacterium enabling the understanding of the physiology and pathogenesis of M.tuberculosis. The pioneering work done by Hatfull and his team has laid foundation for understanding of mycobacteriophages [6].

Mycobacteriophages possess attributes of natural bactericidal agents and specificity. In addition their relative low cost of production makes them appealing as a novel method of therapy [7]. Mycobacteriophages that have a multiple host range, have viral life cycles which allow them to go through lytic cycle, lysogenic cycle, or sometimes a combination of the two. This diversity in their mechanism enables the mycobacteriophages to be used to manipulate the bacteria they are introduced into. This makes them attractive as viralbased antibacterial agents to assist or replace conventional antibiotics. The most common Mycobacterium used to isolate mycobacteriophage is M. smegmatis [5]. As it is hazardous to work with pathogenic bacteria and the incubation period for studying bacteria like M. tuberculosis is much longer, it is beneficial to use a harmless host that replicates quickly such as $M$. smegmatis to isolate phage, and then test the phages obtained for their ability to infect other Mycobacterial species [8].There is a need for inexpensive, rapid and simple diagnostic system for M.tuberculosis, as conventional diagnosis is complicated by the slow growth of the bacteria. The ability of mycobacteriophages to infect mycobacterial hosts specifically and efficiently makes them suitable for use in a diagnostic system [9].For phage therapy to be effective it is necessary to understand phage genomics. This understanding can come about through the addition of phages and their genomes to international databases (8). Hence the objective of this study is to isolate mycobacteriophages using a nonpathogenic strain of Mycobacterium such as M.smegmatis and then testing the resulting novel phages for their capability to infect the $\mathrm{M}$. tuberculosis and other species of Mycobacterium. This would be first step towards exploiting the potential of phages as therapeutic agents. The purpose of this research is to increase knowledge of phage biodiversity by isolating and studying host diversity of novel mycobacteriophages from the environment and to improve our understanding of Mycobacteria spp.

\section{METHODOLOGY}

\section{Cultivation of Mycobacterium smegmatis MTCC 994.}

Mycobacterium smegmatis MTCC 994(IMTECH,

Chandigarh) a non-virulent mycobacterial strain was used as the host for isolation of mycobacteriophage. The host strain was cultivated at $37^{\circ} \mathrm{C}$ in Nutrient broth for $48 \mathrm{hrs}$.

\section{Collection of Soil Sample}

Soil samples from different locations in and around regions of Mumbai and Thane were collected in sterile test tubes.

\section{Isolation of phages:}

$1 \mathrm{gm}$ of soil sample was thoroughly mixed and vortexed with $5 \mathrm{ml}$ of Mycobacteriophage buffer (1M Tris, pH7.5, $1 \mathrm{M}$ $\mathrm{MgSO}_{4}, 4 \% \mathrm{NaCl}, 0.1 \mathrm{M} \mathrm{CaCl}$ )(website 6).The resulting mixture was filtered using 0.22 micron membrane filter. $5 \mathrm{ml}$ of filtered sample was then added to $25 \mathrm{ml}$ of $\log$ phase culture of Mycobacterium smegmatis in Nutrient Broth and incubated at $37^{\circ} \mathrm{C}$. Additional $10 \mathrm{ml}$ of log phase culture was added to the flask for 3 consecutive days. Following the enrichment procedure the growth medium was centrifuged at $2000 \mathrm{~g}$ for $5 \mathrm{~min}$ to remove the cellular debris. The supernatant was then filtered using 0.22 membrane filter and the filtrate obtained was tested for the presence of phages using qualitative plaque method. The filtrate was stored with chloroform at $4^{\mathrm{O}} \mathrm{C}$.

\section{Host Diversity:}

The ability of the isolated mycobacteriophages to infect $M$. fortuitum subsp. fortuitum MTCC 993, Mycobacterium kansasii MTCC 3058, Mycobacterium avium subsp. avium MTCC 1723 and Mycobacterium tuberculosis MTCC 300 was determined by enrichment of each of the mycobacteriophages using each of the above Mycobacterium spp. as host. This was followed by confirming the adaptability to new host by qualitative plaque test with the respective host Mycobacteria spp.

\section{RESUlTS AND DISCUSSION}

A total of 14 soil samples from around different locations of Mumbai and Thane were tested for the presence of Mycobacteriophages using Mycobacterium smegmatis as host. Of the 14 soil samples tested, phages were obtained in 10 samples.The mycobacteriophages obtained were further enriched using four different Mycobacterium spp. as host namely Mycobacterium spp i.e M. fortuitum subsp. fortuitum MTCC 993, Mycobacterium kansasii MTCC 3058, Mycobacterium avium subsp avium MTCC 1723 andMycobacterium tuberculosis MTCC 300.Following enrichment, phages were tested for their ability to infect the 
new host species by qualitative plaque method. The results are tabulated in Table1.

Table 1.Host diversity of the Mycobacteriophages

\begin{tabular}{|c|c|c|c|c|c|}
\hline & \multicolumn{5}{|c|}{ Mycobacterium strains used } \\
\hline $\begin{array}{c}\text { Phage } \\
\text { Sample }\end{array}$ & $\begin{array}{c}\text { M. } \\
\text { Name } \\
\text { MTCC } \\
\mathbf{9 9 4}\end{array}$ & $\begin{array}{c}\text { M. } \\
\text { tuberculos } \\
\text { is } \\
\text { MTCC } \\
300\end{array}$ & $\begin{array}{c}\text { M. } \\
\text { fortuitum } \\
\text { subsp } \\
\text { fortuitum } \\
\text { MTCC } \\
993\end{array}$ & $\begin{array}{c}\text { M. } \\
\text { Mansasii }\end{array}$ & $\begin{array}{c}\text { M. } \\
\text { Avium } \\
\text { subsp. } \\
\text { avium } \\
\text { MTCC } \\
1723\end{array}$ \\
\hline G1 & + & + & + & + & + \\
\hline G2 & + & - & + & + & + \\
\hline G3 & + & - & + & + & + \\
\hline J1 & + & + & + & + & + \\
\hline N1 & + & - & + & - & + \\
\hline N2 & + & - & - & - & + \\
\hline D1 & + & + & + & + & + \\
\hline D2 & + & - & + & - & + \\
\hline D3 & + & - & + & - & + \\
\hline Ga & + & + & - & + & + \\
\hline
\end{tabular}

$\mathrm{Ga}$ is water sample collected fromGanga river (Uttar Pradesh) while all the others were soil samples collected from different regions in Mumbai.

Key: + Plaques seen - No plaques seen

Phagesfrom sample no G1, J1, D1 were found to infect all the 4 different species of Mycobacterium besides Mycobacterium smegmatis. Remaining 7phages was found to infect Mycobacterial species other than Mycobacterium smegmatis but they did not infect all the 4 hosts. Phages G1, J1, D1were purified, concentrated by Polyethylene Glycol 8000(PEG) precipitation and used for further studies. In this study three different mycobacteriophages were isolated, having a wide host range infecting 5 different species of Mycobacterium including Mycobacterium tuberculosis MTCC 300, M. fortuitum MTCC993, Mycobacterium kansasii MTCC 3058, Mycobacterium avium MTCC 1723 and Mycobacterium smegmatis MTCC 994.

These phages could be explored for different practical applications such as therapeutic use or in diagnostic kits. Isolation and characterization of novel mycobacteriophages in this study increases the knowledge of mycobacteriophage diversity which could be of help to better understand Mycobacteria and thus be able to treat mycobacterial infections.

\section{DISCUSSION:}

The first mycobacteriophage was isolated in 1947 by Gardner and Weiser at the University of Washington in Seattle from moist leaf compost samples [10]. Since then more than 9000 phages have been reported using M.smegmatis as host and above 1500 of these are completely sequenced (website 4). A large collection of mycobacteriophages and their sequenced genomes have been reported by the Phage Hunters Integrating Research and Education (PHIRE) program at the University of Pittsburgh, and the Science Education Alliance Phage Hunters Advancing Genomics and Evolutionary Sciences (SEAPHAGES) program by Professor Graham Hatfull and his coworkers from different environmental samples [4]. Stella et al., in 2013 has reported isolation of 18 novel phages from soil and water samples from several places in Argentina. In these phages certain novel characteristics such as propagation at low temperature of $30^{\circ} \mathrm{C}$ have been reported. These plaques were cloned and amplified. On the basis of plaque morphology, nine phages produced clear plaques while nine phages gave turbid plaques. The ability of these isolated mycobacteriophages to infect $M$. fortuitum, $M$. kansasii, M. bovis var BCG and M. tuberculosis H37Rv was also tested. Four mycobacteriophages were able to propagate in M. tuberculosis H37Rv and M. bovis var BCG [6].Hawtrey et al in 2011 reported the isolation of 17 different phages by students of Genome Discovery and Research course, Kentucky(US) from varied soil samples. Several of these phages were completely sequenced, annotated and found to be novel [8].

Rybniker et al in 2006 also tested host range of 14 mycobacteriophages using 8 mycobacterial species. Three of these phages D29, L5 and Bxz2, were found to have a broad host range, and formed plaques on all of the slow-growing Mycobacterium species, except for M.marinum and one strain of M.scrofulaceum. Six mycobacteriophages did not form plaques on any species other than M. smegmatis[11].

\section{CHALLENGES AND LIMITATIONS}

One of the suggested applications using Mycobacteriophages is phage therapy to treat Mycobacterial infections. Although there has been some laboratory testing of phage therapy in M. tuberculosis-infected guinea pigs, no human trials are yet reported. Also there is considerable potential to use phages prophylactically as it can interfere specifically with TB transmission. For example, if a patient is diagnosed with tuberculosis, family members and co-workers in close contact can have phages aspirated into their upper respiratory tract, where these phages could lyse the M. tuberculosis cells that gain entry into the respiratory tract. The phages will prevent $M$. tuberculosis from establishing an infection. As transmission typically involves small numbers of cells, it should be possible to deliver a sufficient amount of phage particles [4]. 
There are certain impediments to phage therapy. Delivery of phages to the lungs should be relatively simple, although there is considerable doubt as to whether they would effectively reach their bacterial hosts, which may be intracellular residing within the granulomas. An alternative way is to use infected surrogate non virulent mycobacterial cells for delivery of phages. Unfortunately, there are relatively few efficient phages available that can kill $M$. tuberculosis. As phage resistance is expected, for efficient killing of M.tuberculosis; a consortium of three to six mycobacteriophages that would elicit different resistance mechanisms in the host should be used. Because only a subset of those phages isolated using M. smegmatis also infect $M$. tuberculosis, isolation of additional phages known to infect M. tuberculosis is desirable [4].

\section{CONCluSionand FUtURE SCOPE}

Mycobacteriophages have several applications. Generalized transduction of genetic markers in M. smegmatis was one of the earliest applications of mycobacteriophages [12]. William R. Jacobs and his team have initiated the construction of novel Escherichia coli-Mycobacterium shuttle vectors using mycobacteriophageTM4 to introduce recombinant DNA into the mycobacteria such as Mycobacterium smegmatis , M. tuberculosis and Mycobacterium bovis BCG (bacilleCalmette-Guerin), by inserting $E$. coli cosmids into nonessential regions of mycobacteriophage DNAs [13].

Mycobacteriophages are extremely diverse in their genetic makeup with many diverging evolutionary path ways. They infect many types of mycobacteria and remain extremely specific to their host range. Each phage goes through lytic and/or lysogenic cycles allowing them to destroy the bacteria they infect. Due to their broad host range and genetic differences each phage cluster has the potential to push through antibiotic resistance barriers [5].

Previously mycobacteriophages were used to differentiate strains of clinical significance and schemes were established to phage type M.avium, M.kansasii and M.xenopi, M.fortuitum and M.tuberculosis. But these techniques have become redundant since the introduction of molecular typing method. The most widely used mycobacteriophage for diagnostic test is D29 due to its wide host range and easy maintenance. A commercial diagnostic kit FAST Plaque TB has been developed using D29. Mycobacteriophages are also used for construction of Luciferase reporter phage using TM4(lytic phage),D29 and L5 as means of detecting viable mycobacteria [14].

Bacteriophages have been engineered towards a wide range of applications including pathogen control, detection and targeted drug delivery. Recent advances in synthetic biology have enabled the development of new molecular biology techniques that could be used to construct specialized bacteriophages with new functionalities [15].

The resulting mycobacteriophages will be genomically characterized, annotated, compared and classified into "Phamilies" of mycobacteriophages. Therefore understanding the biology of mycobacteriophages and their host diversity is key towards understanding the host Mycobacterium spp. This would be first step towards exploiting the potential of phages as therapeutic agents.Thus by contributing to the pool of available phage information, the study will further help with the implementation of phage therapy as an alternative cure to Mycobacterial infections

\section{REFERENCES}

[1]. P.M. Small, "Tuberculosis: a new vision for the $21 \mathrm{st}$ century”,Kekkaku. Vol. 84, Issue. 11, pp.721-726, 2009.

[2]. M.B. Lapenkova , N.S. Smirnova, P.N. Rutkevich,M.A. Vladimirsky, "Evaluation of the Efficiency of Lytic Mycobacteriophage D29 on the Model of M. tuberculosis-Infected Macrophage RAW 264 Cell Line", Bulletin of ExperimentalBiology and Medicine, Vol. 164, Issue.3, pp.344346, 2018.

[3]. G.F. Hatfull, "Complete genome sequences of 138 mycobacteriophages", Journal of Virology, Vol. 86, pp.23822384, 2012a.

[4]. G.F.Hatfull , "Mycobacteriophages: Windows into Tuberculosis", Public Library of Science Pathogens,Vol.10, Issue. 3,2014a.e1003953. http://doi:10.1371/journal.ppat.1003953

[5]. S.A.Morris, "Genetic Diversity of Mycobacteriophages and the Unique Abilities of Cluster K". The Corinthian, Vol. 18, Issue. 1, Article 5, 2017 .http://kb.gcsu.edu/thecorinthian/vol18/iss1/5.

[6]. E.J.Stella, J.J. Franceschelli, S.E. Tasselli, H.R. Morbidoni, "Analysis of novel mycobacteriophages indicates the existence of different strategies for phage inheritance in Mycobacteria", Public Library of Science ONE, Vol.8, Issue. 2 , 2013: e56384.http://doi:10.1371/journal.pone.0056384.

[7]. J. M.Inal, "Phage therapy: a reappraisal of bacteriophages as antibiotics", ArchivumImmunologiae et TherapiaeExperimentalis, Vol.51, pp. 237-244, 2003.

[8]. S. Hawtrey,L. Lovell, R. King, "Isolation, Characterization, and Annotation: The Search for Novel Bacteriophage Genomes", The Journal of Experimental Secondary Science, Vol. 1, Issue. 2, pp. 19, 2011.

[9]. G.F. Hatfull, , “ The secret lives of mycobacteriophages", Advances in Virus Research, Vol.82,pp. 179-288, 2012b.

[10]. G. M. Gardner, R. S.Weiser, "A bacteriophage for Mycobacterium smegmatis", Proceedings of The Society for Experimental Biology and Medicine, Vol. 66, pp. 205-206,1947

[11]. J. Rybniker,S. Kramme, P.L. Small, "Host range of 14 mycobacteriophages in Mycobacterium ulcerans and seven other Mycobacteria including Mycobacterium tuberculosis: application for identification and susceptibility testing", Journal of Medical Microbiology ,Vol.55, pp.37-42, 2006.

[12]. G.F. Hatfull , "Molecular genetics of mycobacteriophages", Microbiology Spectrum, Vol. 2, Issue. 2, pp.1-36, 2014b.http:// doi:10.1128/microbiolspec.MGM2-0032-2013.

[13]. W.Jacobs, S.Snapper, M. Tuckman,B.Bloom,"Mycobacteriophage Vector Systems", Reviews of Infectious Diseases,Vol. 11, pp.S404-S410,1989. http://www.jstor.org/stable/4454877 
[14]. R. McNerney, H. Traore,"Mycobacteriophage and their application to disease control", Journal of Applied Microbiology, Vol. 99, pp.223-233, 2005.

[15]. R.A. Costa, C.Milho, J. Azeredo, D.P. Pires, “Synthetic Biology to Engineer Bacteriophage Genomes. In:,Bacteriophage Therapy", Methods in Molecular Biology, Vol. 1693, Human Press, New York, 2018.

[16]. India: World Health Organization, Regional Office for South-East Asia.Bending the curve - ending TB: Annual report 2017, http://apps.who.int/iris/handle/10665/254762.

[17]. http://www.who.int/gho/tb/en/ (cited on $18^{\text {th }}$ January,2018.)

[18]. http://www.who.int/mediacentre/factsheets/fs104/en/ cited on $19^{\text {th }}$ January 2018)

[19]. http://www.who.int/tb/publications/global_report/Exec_Summary_ 13Nov2017.pdf?ua $=1$ (cited on $21^{\text {st }}$ January 2018)

[20]. http://phagesdb.org/hosts/genera/1/(Mycobacteriophage Genome Database, cited on $16^{\text {th }}$ Januay 2018 .)

[21]. http://phagesdb.org/media/workflow/protocols/pdfs/PHProtocol_P hageBuffer.pdf(cited on $15^{\text {th }}$ January 2016).

\section{AUTHORS PROFILE}

Rajitha Satish: is currently pursuing her Ph.D in Microbiology from SIES College. Her main area of interest is Medical Microbiology. She has 9 years of teaching experience and 5 years of research experience. She is working as lecturer in Department of Microbiology, K.C College.She is also member of Mumbai Immunology group.

Dr Anita Desouza: is Associate Professor at Microbiology dept, SIES College. Biochemistry and enzymology are her forte. She has been guiding M.Sc. students since 1996. She is also a Ph.D guide. She has a number of research publications to her credit. 